\title{
DETERMINACIÓN DE LA CONCENTRACIÓN DE GLIFOSATO EN AGUA MEDIANTE LA TÉCNICA DE INMUNOABSORCIÓN LIGADA A ENZIMAS (ELISA)
}

\author{
Enrique V. PARAVANI ${ }^{1 *}$, María C. SASAL ${ }^{2}$, Silvana M. SIONE ${ }^{3}$, Emmanuel A. GABIOUD $^{2}$, \\ José D. OSZUST ${ }^{3}$, Marcelo G. WILSON ${ }^{2,3}$, Luisina DEMONTE ${ }^{4}$ y María R. REPETTI ${ }^{4}$
}

${ }^{1}$ Facultad de Ingeniería, Universidad Nacional de Entre Ríos. Ruta 11, kilómetro 10.5, Oro Verde, Entre Ríos (3101), Argentina.

${ }^{2}$ Grupo Recursos Naturales y Factores Abióticos, Estación Experimental Agropecuaria Paraná, Instituto Nacional de Tecnología Agropecuaria. Ruta 11, kilómetro 10.5, Oro Verde, Entre Ríos (3101), Argentina.

${ }^{3}$ Facultad de Ciencias Agropecuarias, Universidad Nacional de Entre Ríos. Ruta 11, kilómetro 10.5, Oro Verde, Entre Ríos (3101), Argentina.

${ }^{4}$ Facultad de Ingeniería Química, Universidad Nacional del Litoral. Santiago del Estero 2654 Piso 6. Santa Fe (3000), Argentina.

*Autor para correspondencia: evparavani@bioingenieria.edu.ar

(Recibido septiembre 2015; aceptado enero 2016)

Palabras clave: contaminación, herbicida, cuantificación, inmunoensayo

\section{RESUMEN}

Los herbicidas constituyen fuentes potenciales de contaminación, siendo el agua uno de los componentes del ambiente más vulnerables. El glifosato ( $\mathrm{N}$-fosfonometil glicina) es el herbicida más utilizado a escala mundial. Actualmente, la información referida a su efecto sobre el agua resulta insuficiente, dado fundamentalmente por los altos costos de los análisis de laboratorio. Los objetivos del presente trabajo fueron evaluar el empleo de la técnica de ELISA como una herramienta rápida para el monitoreo de la calidad de aguas y cuantificar la concentración de glifosato por medio de la técnica de UHPLC-MS/MS. Cuando la técnica de ELISA determina valores positivos de glifosato, se requiere la cuantificación mediante la técnica de UHPLC-MS/MS para determinar la concentración exacta de glifosato en dichas muestras. Los resultados sugieren que la técnica de ELISA es factible de ser implementada en programas de monitoreo de aguas, constituyendo un método exploratorio que permite detectar cualitativamente la presencia o ausencia de glifosato.

Key words: contamination, glyphosate, quantification, immunoassay

\begin{abstract}
Herbicides are potential sources of pollution; water being one of the most vulnerable components of the environment. Glyphosate (N-phosphonomethyl glycine) is the most widely used herbicide worldwide. Currently, the information on its effect on water is insufficient, mainly because of the high costs of lab tests. The objectives of this study were to evaluate the use of the ELISA technique as a quick tool for monitoring water quality and to quantify the concentration of glyphosate through the technique of UHPLC-MS/MS. When ELISA positive values determined glyphosate, quantification
\end{abstract}


by UHPLC-MS/MS technique is required to determine the exact concentration of glyphosate in the samples. The results suggest that the ELISA technique is feasible to be implemented in water monitoring programs, providing a screening method that allows detecting qualitatively the presence or absence of glyphosate.

\section{INTRODUCCIÓN}

La contaminación del aire, suelo o agua es un proceso de alteración no deseado que puede ser derivado de la actividad antrópica. Los agroquímicos utilizados en la actividad agropecuaria pueden generar contaminación ambiental, siendo el agua uno de los principales componentes susceptibles de ser contaminado. Se visualizan dos aspectos que ponen en riesgo la calidad de las aguas debido al uso agrícola: 1) el aporte difuso de las fugas de agroquímicos desde agrosistemas por lixiviación, deriva o escurrimiento y 2) el aporte puntual debido a malas prácticas agrícolas, como lavado de equipos pulverizadores en arroyos o disposición de envases. Los sistemas acuáticos son vulnerables a la contaminación al ser los receptores naturales de sustancias y residuos de las actividades agrícolas e industriales (Manahan 2007).

En Argentina, el glifosato es el herbicida de mayor volumen empleado, asociado al uso casi exclusivo de variedades transgénicas de soja (32 millones de ha/ año) y a los tratamientos para barbecho en siembra directa. El volumen de ventas de este producto ha registrado un crecimiento continuo en los últimos años, en 2011 se comercializaron más de 160 millones de kg (CASAFE 2012). La provincia de Entre Ríos, Argentina, no escapa a esta tendencia. La agricultura se está tornando cada vez más especializada y homogénea y se le destinan más de 13 millones de litros de productos fitosanitarios cada año. En este volumen, los herbicidas constituyen el $76 \%$, la mayor proporción aportada por el glifosato, situación derivada del esquema productivo en esta provincia que se caracteriza por la alta presión del cultivo de soja.

El glifosato, al igual que el resto de los fitosanitarios, puede alcanzar los ecosistemas acuáticos por deriva proveniente de aplicaciones cercanas o por escurrimiento durante lluvias intensas (Di Fiori et al. 2012). Estudios de lixiviación y escurrimiento indican que el glifosato puede ser transportado hacia las capas profundas del suelo debido al flujo preferencial por macroporos o hacia cursos de agua superficiales cuando su aplicación se realiza en momentos anteriores a lluvias intensas (Flury 1996, Villholth et al. 2000, Jaynes et al. 2001, Elliot et al. 2002, Petersen et al. 2002, Sasal et al. 2010). En Argentina, la Sub- secretaría de Recursos Hídricos de la Nación publicó en el año 2003, los niveles guía nacionales de calidad de agua ambiente correspondientes a glifosato. Para fuentes de provisión de agua para consumo humano se establece un nivel $\leq 300 \mu \mathrm{g} / \mathrm{L}$, expresado como sal de glifosato con isopropilamina, que corresponde a la protección de la biota acuática un valor crónico final (FCV, por sus siglas en inglés) de $240 \mu \mathrm{g} / \mathrm{L}$. La guía de calidad de agua para la protección de la vida acuática (CWQG, por sus siglas en inglés) publicada por el Ministerio de Ambiente de Canadá, establece como umbral de largo plazo para el agua dulce un valor de $800 \mu \mathrm{g} / \mathrm{L}$ de glifosato (Canadian Council of Ministers of the Environment 2012).

En cursos de aguas superficiales de la provincia de Entre Ríos se ha detectado la presencia de glifosato (Primost 2013, Sasal et al. 2013). Ayarragaray et al. (2014) también han determinado glifosato en arroyos de la provincia de Santa Fe (Argentina) que reciben el agua de escorrentía de los campos agrícolas aledaños. Estos autores concluyen que, luego de las aplicaciones, este herbicida persiste en el ambiente y se degrada, siendo su principal metabolito el ácido aminometilfosfónico (AMPA) y que una vez que ingresa a los cuerpos de agua se asocia a la fracción particulada y se deposita en los sedimentos.

La persistencia, degradación y movilidad del glifosato en suelos y aguas constituyen temas de considerable interés y debate. Si bien se introdujo en el mercado mundial en 1971, la información referida a su impacto ambiental es limitada (Piccolo et al. 1994) debido a que es una molécula muy difícil de analizar. Para la determinación de su concentración es necesaria la derivación de la molécula para su posterior detección por la técnica de cromatografía líquida de alta eficiencia (HPLC, por sus siglas en inglés; Maitre et al. 2008). Peruzzo et al. (2008) registraron concentraciones de glifosato de 100 a $700 \mu \mathrm{g} / \mathrm{L}$ en cuatro cursos de agua del área agrícola de la Pampa Ondulada (Buenos Aires, Argentina). Los autores encontraron una relación directa entre la dosis de aplicación y la concentración en agua superficial y sedimentos. Investigaciones desarrolladas en cursos de agua superficial de la provincia de Santa Fe (Argentina) asociados a ambientes agrícolas, han informado niveles de glifosato y AMPA en la fracción disuelta, 
que varían entre $0.5 \leq 7.8 \mu \mathrm{g} / \mathrm{L}$ y $0.5 \leq 3.4 \mu \mathrm{g} / \mathrm{L}$, respectivamente. En los sedimentos, las concentraciones registradas variaron entre $8.0 \leq 49.1 \mu \mathrm{g} / \mathrm{kg}$ húmedo y $11.2 \leq 60.7 \mu \mathrm{g} / \mathrm{kg}$ húmedo para glifosato y AMPA, respectivamente (Ayarragaray et al. 2014).

El estudio del impacto ambiental de tecnologías agrícolas es incipiente en Argentina. Trabajos realizados en la provincia de Entre Ríos han arrojado resultados preliminares sobre el contenido de glifosato en suelos (Maitre et al. 2008). Se ha avanzado en la cuantificación de este herbicida en aguas mediante análisis químicos (Díaz et al. 2008, Sasal et al. 2010) y bioensayos (Lallana et al. 2008). Sin embargo, resulta insuficiente la información referida al efecto que genera la aplicación de este agroquímico en los agrosistemas y en aguas de escorrentía. Esto se debe fundamentalmente a los elevados costos de los análisis de laboratorio, que impiden llevar adelante un programa de monitoreo.

El glifosato y el AMPA son compuestos poco volátiles y altamente hidrófilos (Corbera 2007). Para su cuantificación en agua se desarrolla un proceso de extracción, concentración y análisis de la muestra. El ensayo HPLC constituye uno de los métodos analíticos más difundidos para la determinación de glifosato y AMPA en agua, dado el carácter iónico que presentan estas sustancias (Nedelkoska y Low 2004). Sin embargo, la aplicación de esta técnica se ve limitada por sus elevados costos y por la necesidad de disponer de una adecuada infraestructura.

Actualmente, se ha desarrollado una técnica alternativa al HPLC para la determinación del plaguicida en agua, conocida como ensayo por inmunoadsorción ligado a enzimas (ELISA, por sus siglas en inglés) con el equipo Glyphosate Magnetic Particle Kit, Abraxis ${ }^{\circledR}$. Esta técnica se fundamenta en la interacción del analito con un anticuerpo que es reconocido por su alta afinidad y especificidad. Se trata de un método simple, de bajo costo, rápido, sensible y selectivo. Además, no requiere de instrumentación sofisticada y permite analizar un elevado número de muestras en corto tiempo.

Los objetivos del presente trabajo fueron determinar la concentración de glifosato en agua de escorrentía en sistemas agrícolas mediante las técnicas de ELISA y de cromatografía líquida de ultra alta eficiencia acoplada a espectrometría de masa en tándem (UHPLC-MS/MS, por sus siglas en inglés) y analizar la viabilidad de la primer técnica para su implementación en programas de monitoreo de calidad del agua. La información generada servirá de base para futuros estudios acerca del impacto que tiene el glifosato sobre el agua de escorrentía. Asimismo, aportará datos para esclarecer percepciones de riesgo que se han generalizado tanto en la población rural como en la urbana y periurbana de la provincia de Entre Ríos (Argentina).

\section{MATERIALES Y MÉTODOS}

\section{Sitio de estudio}

El estudio se desarrolló en la Estación Experimental Agropecuaria del Instituto Nacional de Tecnología Agropecuaria (INTA) de la ciudad de Paraná (31 50 '47.44" sur, 60031'48.5" oeste), ubicada en Entre Ríos, Argentina (Fig. 1).

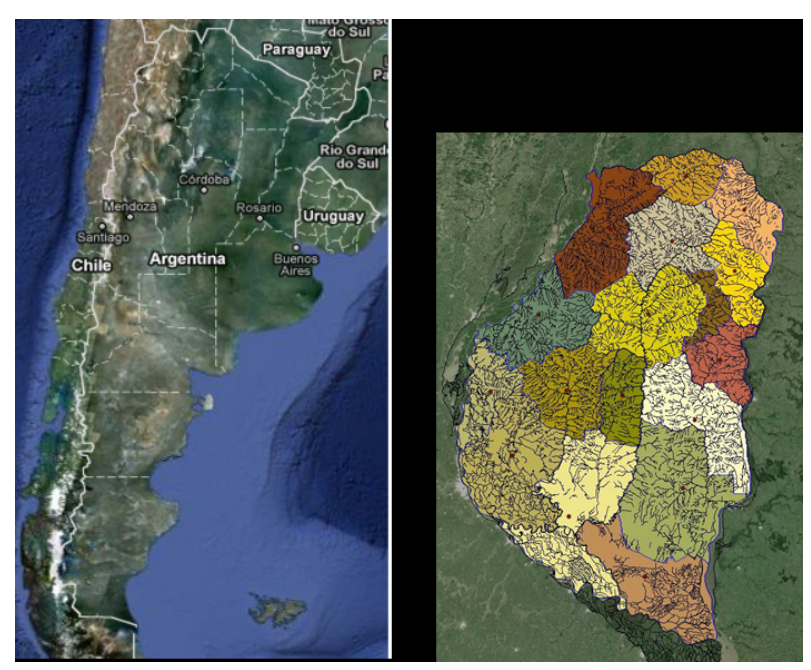

Fig. 1. Sitio de estudio. Localización de la Estación Experimental, Instituto Nacional de Tecnología Agropecuaria (INTA), Paraná, Entre Ríos, Argentina

\section{Recolección de muestras}

En el periodo julio de 2009 - noviembre de 2012 fueron recolectadas 93 muestras de agua de escorrentía en sistemas agrícolas (Cuadro I): 36 obtenidas mediante la utilización de un simulador de lluvia (Sasal et al. 2015; Fig. 2) y 57 en parcelas de escorrentía (con diferentes secuencias de cultivo) recolectadas a posteriori de eventos de precipitación que generan escurrimientos con lluvia natural (Sasal et al. 2010; Fig. 3).

Las muestras se recogieron en recipientes de plástico de policloruro de vinilo (PVC) de $200 \mathrm{~mL}$ de color ámbar, previamente lavados con ácido clorhídrico acuoso $(\mathrm{pH} 2)$ y repetidamente enjuagados con agua desionizada. Durante el muestreo los recipientes fueron lavados dos veces con el agua a muestrear y posteriormente fueron tomadas las muestras, mismas que se rotularon y almacenaron a $-20^{\circ} \mathrm{C}$ en condiciones de oscuridad hasta el momento de su análisis. 
CUADRO I. FECHA DE EXTRACCIÓN, NÚMERO Y ORIGEN DE MUESTRAS DE AGUA DE ESCORRENTÍA

\begin{tabular}{lcc}
\hline Fecha de extracción & Número de muestras & Origen \\
\hline Julio 2010 & 5 & $\mathrm{PE}$ \\
Noviembre 2010 & 6 & $\mathrm{PE}$ \\
Diciembre 2010 & 8 & $\mathrm{PE}$ \\
Enero 2011 & 13 & $\mathrm{PE}$ \\
Marzo 2011 & 21 & $\mathrm{PE}$ \\
Mayo 2011 & 4 & $\mathrm{PE}$ \\
Noviembre 2012 & 36 & $\mathrm{SL}$ \\
Total de muestras & 93 & \\
\hline
\end{tabular}

PE: parcela de escorrentía (lluvia natural), SL: simulación de lluvia

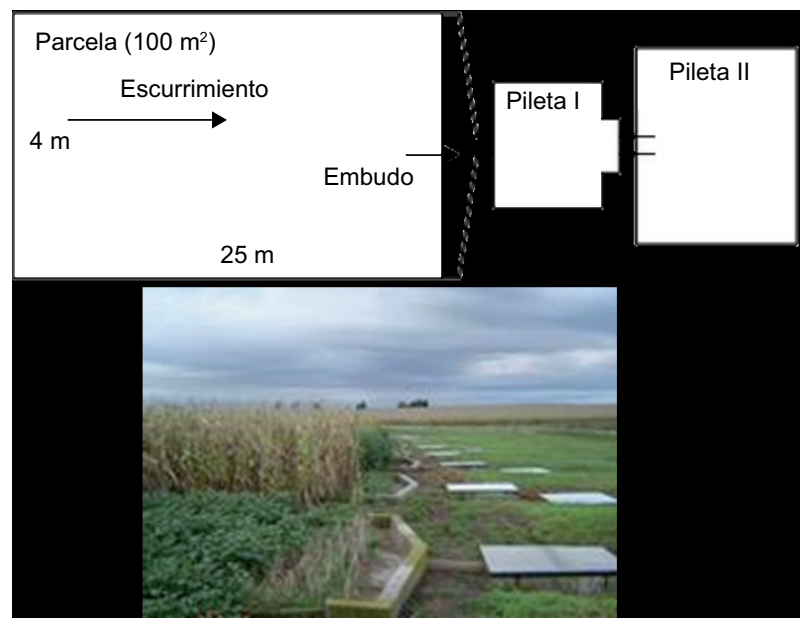

Fig. 2. Detalle de las parcelas de escorrentía

\section{Determinación de la concentración de glifosato mediante la técnica de ELISA}

El equipo ELISA de la empresa Abraxis ${ }^{\circledR}$ para la determinación de glifosato aplica el principio del inmunoensayo competitivo, con la derivación previa de la muestra (Cailla et al. 1973). Una característica importante del ensayo es que se llevan a cabo de manera simultánea dos pasos clave del proceso: primero, el analito derivado se preincuba con el anticuerpo específico para glifosato y segundo, este último se une a anticuerpos de inmunoglobulina (IgG) inmovilizados sobre la superficie sólida de los tubos donde se lleva a cabo el ensayo. La proporción de anticuerpos específicos a glifosato que permanecen libres en la superficie sólida reaccionan con el glifosato conjugado a la enzima, cuya actividad es medida por su sustrato colorimétrico. La preparación de los reactivos fue llevada a cabo según las instrucciones del proveedor. Para realizar la derivación, a $50 \mu \mathrm{L}$ de muestra se le

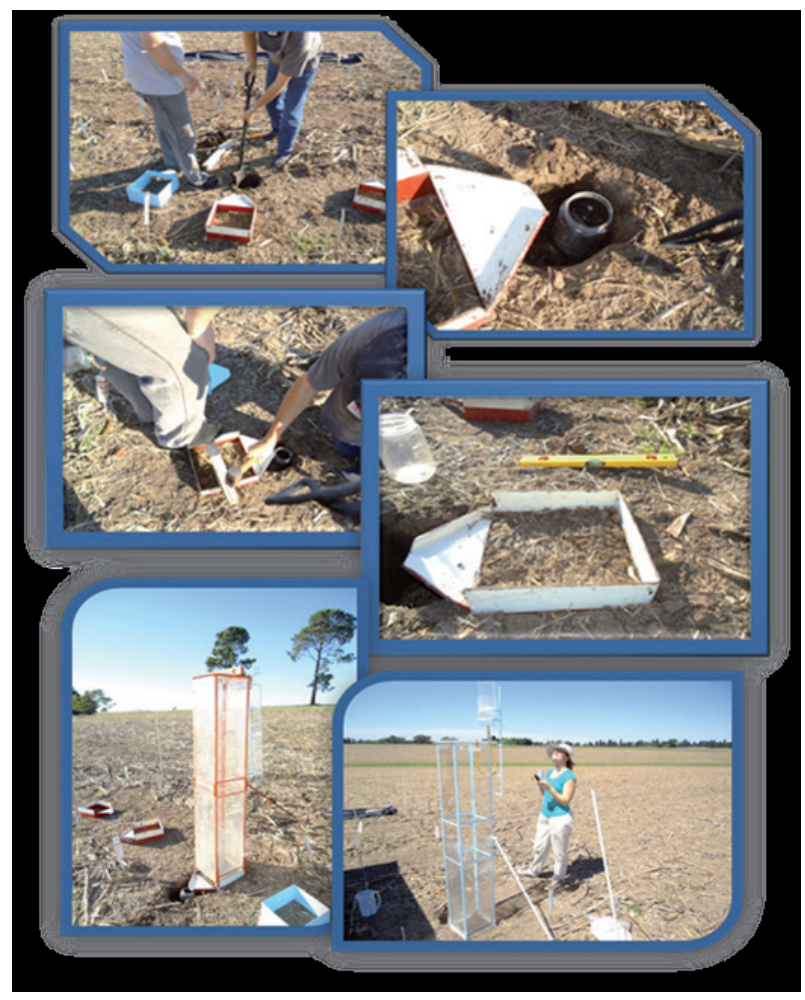

Fig. 3. Instalación y funcionamiento del Simulador de lluvia portátil

agregaron $200 \mu \mathrm{L}$ de solución de ensayo y $20 \mu \mathrm{L}$ de reactivo de derivación. Posteriormente se mezcló y se incubó a temperatura ambiente durante $10 \mathrm{~min}$. Luego de la derivación, las partículas paramagnéticas unidas al anticuerpo específicos antiglifosato fueron agregadas. La enzima conjugada a glifosato fue añadida a continuación, instante en el que fue producida una reacción competitiva entre el glifosato que puede estar en la muestra y la enzima conjugada. Al final del periodo de incubación se aplicó un campo magnético para retener en el tubo de ensayo las partículas paramagnéticas. Tras la decantación, las partículas fueron lavadas con solución de lavado dos veces. La presencia de glifosato fue detectada con la adición de la solución de color (azul celeste) que contiene el sustrato enzimático y el reactivo cromógeno incubados $20 \mathrm{~min}$. El marcado con la enzima unida al anticuerpo antiglifosato cataliza la conversión de la mezcla sustrato/cromógeno a un producto coloreado (amarillo). Después de incubar 20 min, la reacción fue detenida con una solución para ese fin. El color desarrollado $(450 \mathrm{~nm})$ es inversamente proporcional a la concentración de glifosato en dicha muestra. Para la determinación de las absorbancias se empleó un espectrofotómetro marca Metrolab ${ }^{\circledR}$, modelo Visible 1600 plus (Buenos Aires, Argentina). 
Las curvas de calibración se establecieron con soluciones patrón proporcionadas por Abraxis ${ }^{\circledR}$ en cuatro niveles de concentración, comprendidos entre 0.075 y $4 \mu \mathrm{g} / \mathrm{L}(0.075,0.2,1$ y $4 \mu \mathrm{g} / \mathrm{L})$, con tres repeticiones cada una. También se utilizó una solución de calidad analítica $(0.5 \mu \mathrm{g} / \mathrm{L})$, empleada como testigo interno de las soluciones patrón.

Se calculó la regresión lineal para determinar la existencia de relación entre los valores de absorbancia y las concentraciones de glifosato en las muestras de agua. Concentraciones de glifosato inferiores al primer patrón $(0.075 \mu \mathrm{g} / \mathrm{L})$ fueron consideradas como no detectadas (ND), mientras que valores mayores al último patrón $(4 \mu \mathrm{g} / \mathrm{L})$ se tomaron como concentraciones no cuantificables (NC) por el ensayo, por lo que tuvieron que diluirse para su posterior cuantificación. Todas las determinaciones de la concentración de glifosato se realizaron por triplicado.

\section{Determinación de la concentración de glifosato por la técnica de UHPLC-MS/MS}

La determinación de glifosato por UHPLC-MS/ MS fue realizada en el Laboratorio del Programa de Investigación y Análisis de Residuos y Contaminantes Químicos (PRINARC, Santa Fe, Argentina) de acuerdo con Ibañez et al. (2006) y Hanke et al. (2008), con modificaciones. Se trabajó con un volumen de muestra de $3 \mathrm{~mL}$, previamente se efectuó la homogeneización y filtración con un filtro de membrana de celulosa de $0.45 \mu \mathrm{m}$. Posteriormente las muestras fueron colocadas en tubos de centrífuga de teflón de $15 \mathrm{~mL}$ de capacidad, a las que se les agregó ácido clorhídrico $6 \mathrm{M}$ a pH 1 (para una máxima disociación del complejo analito-catión). Luego, las muestras fueron marcadas con glifosato 2-C13 N15 y AMPA C13 N15 como patrón interno ( $40 \mu \mathrm{L}$ de ILS de $1 \mathrm{mg} / \mathrm{L}$ ) y neutralizadas a $\mathrm{pH} 7$ con hidróxido de potasio $6 \mathrm{M}$. Los pasos de la derivación fueron iniciados con la adición de $0.5 \mathrm{~mL}$ de amortiguador borato (pH 9), 9-fluorenilmetilcloroformato (FMOC-Cl, 6 $\mathrm{g} / \mathrm{L})$ y acetonitrilo $(\mathrm{MeCN})$. Después de la reacción durante toda la noche $(12 \mathrm{~h})$ a temperatura ambiente $\left(22^{\circ} \mathrm{C}\right)$, el paso de derivación fue inactivado con ácido fórmico (AF) a $\mathrm{pH} 3$. Posterior a la filtración con un filtro de celulosa de $0.45 \mu \mathrm{m}$, el extracto derivado fue colocado en un cartucho Oasis HLB (200 mg, Waters, Milford, MA, EUA). Los cartuchos fueron previamente acondicionados con $5 \mathrm{~mL}$ de $\mathrm{MeOH}$ (alcohol metílico, calidad óptima, Fisher Scientific, EUA), seguido por $5 \mathrm{~mL}$ de AF (99\% pureza, Tedia Company INC, EUA) al $0.1 \%(\mathrm{v} / \mathrm{v})$. Los eluídos fueron descartados y el cartucho fue lavado con $3.5 \mathrm{~mL}$ de diclorometano (98\% pureza, Ciccarelli,
Argentina). El analito fue eluído del cartucho con 9 $\mathrm{mL}$ de $\mathrm{MeOH}$. La alícuota alcohólica fue evaporada y reconstituida con la fase móvil $\mathrm{H}_{2} \mathrm{O} / \mathrm{MeCN}$ (98:2 $+0.1 \% \mathrm{AF}$ ). A continuación se filtró con jeringa de nylon de $0.2 \mu \mathrm{m}$ y los filtrados se inyectaron para su análisis.

El análisis fue realizado por UHPLC-MS/MS, con un cromatógrafo Acquity UPLC (The Science of What's Possible ${ }^{\circledR}$, Waters) acoplado a un espectrómetro de masa de triple cuadrupolo con fuente ESI (TQD, Waters Micromass, Reino Unido). Las separaciones se realizaron a $40{ }^{\circ} \mathrm{C}$ usando una columna Acquity UPLC HSS C18 (tamaño de partícula $1.8 \mu \mathrm{m}, 2.1 \times 100 \mathrm{~mm}$ ) a una velocidad de flujo de $0.35 \mathrm{~mL} / \mathrm{min}$. Las alícuotas de $10 \mu \mathrm{L}$ del patrón y los extractos de las muestras fueron introducidas por medio de un inyector automático (Waters). La fase móvil consistió de $\mathrm{H}_{2} \mathrm{O} / \mathrm{MeCN}(98: 2+0.1 \% \mathrm{AF}$, denominada $\mathrm{A} 1)$ y $\mathrm{MeCN}(0.1 \% \mathrm{AF}$, denominada $\mathrm{B} 1)$, operando con un gradiente de tiempo de ejecución de 10 min. La ionización fue realizada en modo de ion positivo usando nitrógeno como gas de desolvatación y gas de cono a 600 y $15 \mathrm{~L} / \mathrm{h}$, respectivamente. Se aplicó un voltaje de capilar de $1 \mathrm{KV}$ y la temperatura de la fuente fue de $140{ }^{\circ} \mathrm{C}$. La temperatura a la cual se realizó la conversión del aerosol en partículas de sal fue de $500{ }^{\circ} \mathrm{C}$. El gas argón $\left(1.32 \times 10^{-5} \mathrm{mbar}\right)$ fue utilizado en la celda de colisión para producir los iones y la adquisición se realizó en modo de monitoreo de reacción múltiple (MRM, por sus siglas en inglés). Para la cromatografía y la gestión de datos de espectrometría de masa fue empleado el programa MassLynx v 4.1 (The Science of What's Possible ${ }^{\circledR}$, Waters). Todos los otros suministros utilizados fueron de calidad analítica. Para el análisis cuantitativo se realizaron curvas de calibrado con estándares en los siguientes niveles de concentración: $0.1,0.5$, 1, 10, 100 y $500 \mu \mathrm{g} / \mathrm{L}$. Los límites de detección y cuantificación se estimaron como 3 y 10 veces la relación señal/ruido a partir de cromatogramas de solución estándar en el punto más bajo de la curva de calibrado, resultando en las muestras de agua de $0.2 \mu \mathrm{g} / \mathrm{L}$ y $0.6 \mu \mathrm{g} / \mathrm{L}$ respectivamente.

\section{RESULTADOS Y DISCUSIÓN}

\section{Calibración de la técnica de ELISA}

La técnica competitiva de ELISA proporcionó una curva estándar lineal de pendiente negativa con el aumento de la concentración de glifosato (Fig. 4).

Los valores extremos de concentración de glifosato presentaron mayores desviaciones estándar 


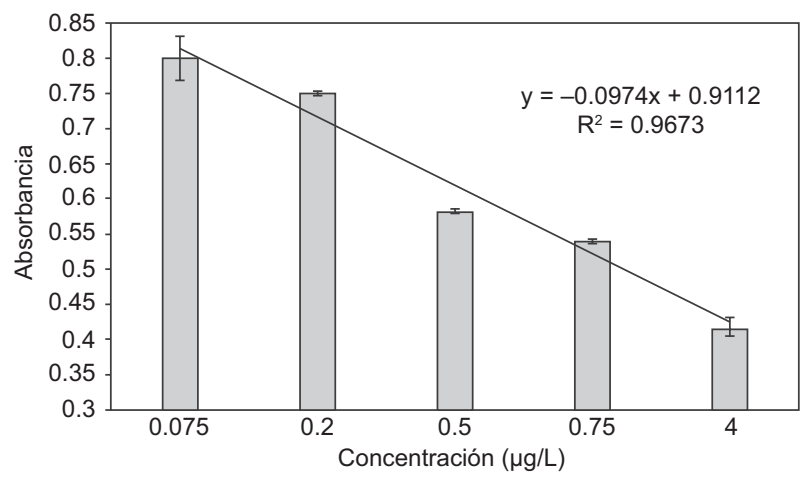

Fig. 4. Curva de calibración estándar para el ensayo ELISA competitivo. Absorbancia vs. concentración de glifosato de los estándares internos y el testigo

$(0.800 \pm 0.032 \mu \mathrm{g} / \mathrm{L}$ y $0.418 \pm 0.013 \mu \mathrm{g} / \mathrm{L})$ respecto a las concentraciones intermedias. El estudio de linealidad de la técnica permitió detectar una correlación positiva entre las absorbancias y las concentraciones del patrón de glifosato $\left(\mathrm{R}^{2}=0.9673\right)$, además de un muy buen ajuste del patrón interno de $0.5 \mu \mathrm{g} / \mathrm{L}$, respecto al resto de las concentraciones patrón.

\section{Determinación de la concentración de glifosato en muestras de agua}

El $61.3 \%$ de las muestras de agua analizadas mediante la técnica ELISA arrojó concentraciones de glifosato inferiores a $0.075 \mu \mathrm{g} / \mathrm{L}$, consideradas como ND. De las 36 muestras que resultaron positivas para los parámetros cuantificables por esta técnica, 11 (31 $\%$ ) presentaron concentraciones superiores a $4 \mu \mathrm{g} / \mathrm{L}$ (NC), las que fueron diluidas para su cuantificacion por la técnica de ELISA y posterior confirmacion por UHPLC-MS/MS.

La aplicación de UHPLC-MS/MS permitió detectar glifosato en las 36 muestras de agua que habían resultado positivas con la técnica de ELISA, sin haberse detectado el herbicida en el resto de las muestras analizadas.

El $100 \%$ de las muestras de agua que resultaron positivas para la técnica de ELISA también fueron positivas para la de UHPLC-MS/MS. Por su parte, todas las muestras en las que no fue detectada la presencia de glifosato por UHPLC-MS/MS, resultaron negativas para la técnica ELISA. Del total de muestras de agua positivas por la técnica de UHPLC-MS/ MS, nueve presentaron concentraciones superiores a $4 \mu \mathrm{g} / \mathrm{L}$, tratándose de las mismas muestras que requirieron dilución previa para su análisis mediante la técnica ELISA.

Considerando los diferentes límites de detección, no fue posible efectuar una correlación entre los valores de concentración de glifosato determinados por ambas técnicas. Corbera (2007) indica que la técnica de ELISA es considerada una técnica exploratoria, no confirmatoria de la concentración de glifosato.

Existen diversos trabajos que evalúan la confiabilidad de los resultados obtenidos con la técnica de ELISA frente a la técnica de UHPLC-MS/MS, empleada como una técnica analítica de referencia. Byer et al. (2008) han obtenido una correlación entre la técnica de ELISA y la cromatografía líquida acoplada a espectrometría de masa en tándem (LC-MS/MS, por sus siglas en inglés) con un $\mathrm{R}^{2}=0.88$, mientras que Sanchís et al. (2011) obtuvieron una correlación con un $\mathrm{R}^{2}=0.90$. Sin embargo, en este trabajo no fue posible descubrir una correlación entre ambas técnicas por la diferencia en los límites de detección de ambas, fundamentalmente del equipo de UHPLCMS/MS (Límite de detección $=0.2 \mu \mathrm{g} / \mathrm{L}$ y Límite de cuantificación $=0.6 \mu \mathrm{g} / \mathrm{L})$ con el que se cuantificaron las muestras positivas por ELISA (Fig. 5).

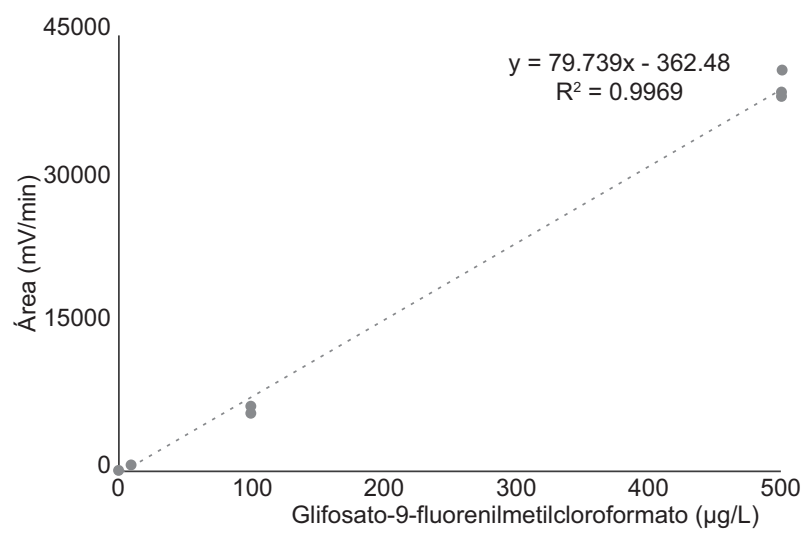

Fig. 5 Curva de calibración para el equipo de cromatografía líquida de ultra alta eficiencia acoplada a espectrometría de masa en tándem

Las muestras de agua que resultaron positivas arrojaron concentraciones de glifosato entre $18.6 \mathrm{y}$ $1552.9 \mathrm{\mu g} / \mathrm{L}$ (UHPLC-MS/MS). En la figura $6 \mathrm{se}$ exponen las frecuencias porcentuales de los rangos de concentración del herbicida en las muestras que resultaron positivas. La mayor frecuencia $(58.3 \%)$ correspondió al rango 0.2 - $100 \mu \mathrm{g} / \mathrm{L}$. Sólo en el 10.4 $\%$ (cuatro muestras) se detectaron valores superiores a los $800 \mu \mathrm{g} / \mathrm{L}$, límite establecido por el Ministerio de Ambiente de Canadá (2012) para la protección de la vida acuática.

Las mayores concentraciones de glifosato fueron registradas con simulaciones de lluvia de alta intensidad, inmediatamente posteriores a las aplicaciones del herbicida (Sasal et al. 2015). 


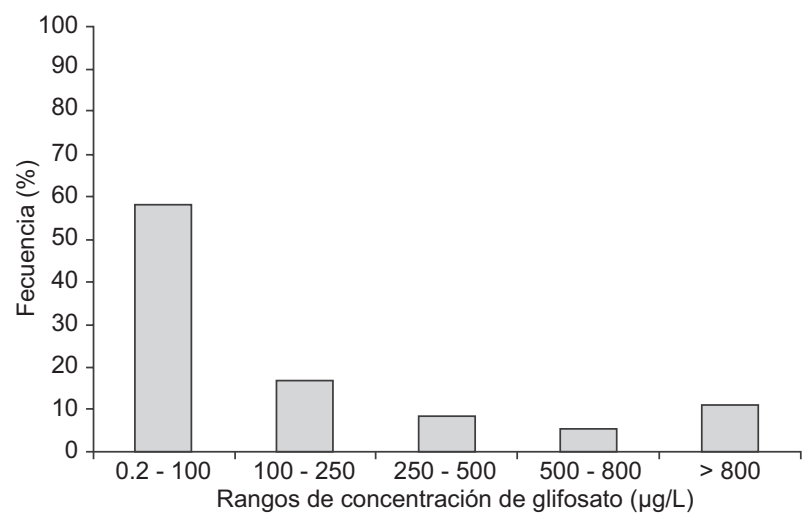

Fig 6. Frecuencia porcentual de rangos de concentración de glifosato por cromatografía líquida de ultra alta eficiencia acoplada a espectrometría de masa en tándem en agua de escorrentía

\section{CONCLUSIONES}

En el presente trabajo se ha logrado reproducir los parámetros estandarizados por los fabricantes del ensayo de ELISA para la determinación de la concentración de glifosato en muestras de agua. Ha sido posible analizar un gran número de muestras y obtener resultados confiables con este método de análisis cualitativo.

El análisis de agua de escorrentía en sistemas agrícolas reveló la presencia de glifosato en bajas concentraciones, inferiores a $250 \mu \mathrm{g} / \mathrm{L}$ en el $75 \%$ de las muestras. Los resultados obtenidos sugieren que es factible la implementación de la técnica de ELISA en programas de monitoreo de la calidad del agua, al ser un método de carácter exploratorio que permite detectar en forma cualitativa la presencia o ausencia de glifosato de forma rápida y apropiada. Sin embargo, cuando esta técnica detecta glifosato, se requiere la aplicación de la técnica de UHPLC-MS/MS para la cuantificación de la concentración del herbicida.

\section{REFERENCIAS}

Ayarragaray M., Regaldo L., Reno U. y Gutiérrez M. F. (2014). Monitoreo de glifosato y ácido aminometilfosfónico (AMPA) en ambientes acuáticos cercanos a la ciudad de San Justo (Santa Fe, Argentina). Memoria. V Congreso Argentino de la Sociedad de Toxicología y Química Ambiental SETAC Argentina. Neuquén, Argentina. 22 al 25 de octubre, 2014. CD-ROM.

Byer J. D., Struger J., Klawunn P., Todd A. y Sverko E. (2008). Low cost monitoring of glyphosate in surface waters using the ELISA method: an evaluation. Environ. Sci. Technol. 42, 6052-6057.

DOI: $10.1021 / \mathrm{es} 8005207$
Cailla H. L., Racine-Welsbuch M. S. y Delange M. N. (1973). Adenosine 3',5' cyclic monophosphate assay at 10-15 mole level. Anal. Biochem. 56, 394-407. DOI: 10.1016/0003-2697(73)90205-4

CASAFE (2012). Cámara de Sanidad Agropecuaria y Fertilizantes [en línea] http://www.casafe.org/publicaciones/estadisticas/ 01/05/2015.

Canadian Council of Ministers of the Environment (2012). Canadian water quality guidelines for the protection of aquatic life: Glyphosate. En: Canadian environmental quality guidelines, Canadian Council of Ministers of the Environment, Winnipeg, $10 \mathrm{pp}$. [en línea] http:// ceqg-rcqe.ccme.ca/download/en/182 15/06/2015

Corbera M. (2007). Desenvolupament de metodologia analitica per a la determinació de glifosat i adjuvants. Tesis Doctoral, Universitat de Girona. Departamento de Química, Unitat de Química Analítica, Girona, España, 208 pp.

Díaz E. L. (2008). Evaluación de residuos de plaguicidas en suelos y aguas cultivados con arroz en Entre Ríos. Memorias. XXI Congreso Argentino de la Ciencia del Suelo. San Luis, Argentina. 13 al 16 de mayo, 2008. CD-ROM.

Di Fiori E., Pizarro H., dos Santos Alfonso M. y Cataldo D. (2012). Impact of the invasive mussel Limnoperma fortunei on glyphosate concentration in wáter. Ecotoxicol. Environ. Saf. 81, 106-13.

DOI: 10.1016/j.ecoenv.2012.04.024

Elliott J. A., Cesna A. J., Best K. B., Nicholaichuk W. y Tollefson L. C. (2002). Leaching ratesand preferential flow of selected herbicides through tilled and untilled soil. J. Environ. Qual. 29, 1650-1656. DOI: $10.2134 /$ jeq2000.00472425002900050036x

Flury M. (1996). Experimental evidence of transport of pesticides through field soil. A review. J. Environ. Qual. 25, 25-45.

Hanke I., Singer H. y Hollender J. (2008). Ultratrace-level determination of glyphosate, aminomethylphosphonic acid and glufosinate in natural waters by solid-phase extraction followed by liquid chromatography-tandem mass spectrometry: performance tuning of derivatization, enrichment and detection. Anal. Bioanal. Chem. 391, 2265-2276.

DOI: $10.1007 / \mathrm{s} 00216-008-2134-5$

Ibanez M., Pozo O. J., Sancho J. V., López F. J. y Hernández F. (2006). Re-evaluation of glyphosate determination in water by liquid chromatography coupled to electrospray tandem mass spectrometry. J. Chromatogr. 1134, 51-55.

DOI: 10.1016/j.chroma.2006.07.093

Jaynes D. B., Ahmed S. I., Kung K. J. S. y Kanwar R. S. (2001). Temporal dynamics for preferential flow to a subsurface drain. Soil Sci. Soc. Am. J. 65, 1368-1376. DOI: $10.2136 /$ sssaj2001.6551368x 
Lallana M. del C., Billard C. E., Elizalde J. H. I. y Lallana V. H. (2008). Bioensayo de germinación de Lactuca sativa (L.): determinación de calidad de agua en represas para riego. Revista Facultad de Ciencias Agropecuarias Universidad Nacional de Cuyo. 40, 29-38.

Maitre M. I., Lorenzatti E. A., Lorenzon M. A. y Enrique S. N. (2008). Adsorción-desorción de glifosato en dos suelos argentinos. Natura Neotropicalis 39, 19-31.

Manahan S. (2007). Introducción a la química ambiental. Ed. Reverté. Barcelona, España, 725 pp.

Nedelkoska T. V. y Low G. K. (2004). High performance liquid chromatography determination of glyphosate in water and plant material after pre-column derivatisation with 9-fluorenylmethyl chloropormate. Anal. Chim. Acta. 511, 145-153.

Peruzzo P. J., Porta A. A. y Ronco A. E. (2008). Levels of glyphosate in surface waters, sediments and soils associated with direct sowing soybean cultivation in North pampasic region of Argentina. Environmental Pollution. 156, 61-66.

DOI: $10.1016 /$ j.envpol.2008.01.015

Petersen C., Holm J., Koch C. B., Jensen H. E. y Hansen S. (2002). Movement of pendimethalin, ioxynil and soil particles to field drainage tiles. Pest Manag. Sci. 59, 85- 96. DOI: $10.1002 /$ ps.609

Piccolo A., Celano G., Arienzo M. y Mirabella A. J. (1994). Adsoption and desorption of glyphosate in some european soils. Environ. Sci. Health. 29, 1105.

DOI: $10.1080 / 03601239409372918$

Primost J. (2013). Estudio de niveles ambientales de glifosato y AMPA en una zona modelo de intensa actividad agrícola en los alrededores de Urdinarrain,
Entre Ríos. Memoria. V Congreso Latinoamericano de Agroecología. La Plata, Argentina. 6 al 9 octubre, 2013. CD-ROM.

Sasal M. C., Andriulo A. E., Wilson M. G. y Portela S. I. (2010). Pérdidas de glifosato por drenaje y escurrimiento en Molisoles bajo siembra directa. Información Tecnológica 21, 135-142.

DOI: 10.1612/inf.tecnol.43741it.09

Sasal M. C., Castiglioni M. G. y Wilson M. G. (2010). Effect of crop sequences on soil properties and runoff on natural rainfall erosion plots under no tillage. Soil Till. Res. 108, 24-29.

DOI: 10.1016/j.still.2010.03.010

Sasal M. C., Demonte L., Cislaghi A., Gabioud E. A., Oszust J. D., Wilson M. G., Michlig N., Beldoménico H. R. y Repetti M. R. (2015). Glyphosate loss by runoff and its relationship with phosphorous fertilization. J. Agric. Food Chem. 63, 4444-4448.

DOI: $10.1021 / \mathrm{jf505533r}$

Sanchís J., Kantiani L., Llorca M., Rubio F., Ginebreda A., Fraile J., Garrido T. y Farré M. (2011). Determination of glyphosate in groundwater samples using an ultrasensitive immunoassay and confirmation by on-line solid-phase extraction followed by liquid chromatography coupled to tandem mass spectrometry. Anal. Bioanal. Chem. 402, 2335-2345.

DOI: $10.1007 / \mathrm{s} 00216-011-5541-y$

Villholth K. G., Jarvis N. J., Jabobson O. H. y de Jonge H. (2000). Field investigations and modeling of particlefacilitated pesticide transport in macroporous soil. J. Environ. Qual. 29, 1298-1309. DOI: $10.2134 /$ jeq2000.00472425002900040037x 